Post-print of Maestro, M. et al. «New high resolution Random Telegraph Noise (RTN) characterization method for resistive RAM» in Solid-state electronics (Elsevier), Vol. 115, Part B (January 2016), p. 140-145. The final version is available at DOI $\underline{10.1016 / j . s s e .2015 .08 .010}$

\title{
NEW HIGH RESOLUTION RANDOM TELEGRAPH NOISE (RTN) CHARACTERIZATION METHOD FOR RESISTIVE RAM.
}

\author{
M.Maestro ${ }^{1}$, J.Diaz ${ }^{1}$, A.Crespo-Yepes ${ }^{1}$, M.B.Gonzalez ${ }^{2}$, J.Martin-Martinez ${ }^{1}$, \\ R.Rodriguez ${ }^{1}$, M.Nafria ${ }^{1}$,F.Campabadal ${ }^{2}$ and X.Aymerich ${ }^{1}$ \\ 1 - Department of Electronic Engineering, Universitat Autònoma de Barcelona (UAB) \\ 08193, Bellaterra (Barcelona), Spain. \\ marcos.maestro@uab.es \\ 2 - Institut de Microelectronica de Barcelona, IMB-CNM (CSIC), \\ 08193, Bellaterra (Barcelona), Spain.
}

\begin{abstract}
Random Telegraph Noise (RTN) is one of the main reliability problems of resistive switching-based memories. To understand the physics behind RTN, a complete and accurate RTN characterization is required. The standard equipment used to analyse RTN has a typical time resolution of $\sim 2 \mathrm{~ms}$ which prevents evaluating fast phenomena. In this work, a new RTN measurement procedure, which increases the measurement time resolution to $2 \mu \mathrm{s}$, is proposed. The experimental set-up, together with the recently proposed Weighted Time Lag (W-LT) method for the analysis of RTN signals, allows obtaining a more detailed and precise information about the RTN phenomenon.
\end{abstract}

\section{Keywords}

Resistive Switching; Random Telegraph Noise; resolution, time constants, RRAM.

\section{Main Text}

\section{INTRODUCTION}

Resistive Random Access Memories (RRAMs) are one of the most promising candidates to replace the current flash memories due to their low power operation, fast switching and great scalability [1-8]. In these devices, typically composed of MIM or 
MIS structures, the resistance of the dielectric can be switched between a low resistance state (LRS) and a high resistance state (HRS), by applying suitable voltages at the device electrodes. Despite their excellent characteristics, RRAMs present some reliability problems, such as the occurrence of current fluctuations in the form of Random Telegraph Noise (RTN) [9-13]. RTN can be observed at both resistance states [14], although RTN is more relevant in the HRS because of the low current through the dielectric $[15,16]$. Since RTN has a major contribution to the device current, its spectra can be crucial to determine the memory window and the correct memory cell performance. Therefore, a precise characterization of the RTN fluctuations in emerging RRAM cells becomes necessary. Until now, standard characterization equipment has been commonly used to characterize RTN, such as semiconductor parameter analyzers (SPAs) with a measuring time resolution of $\sim 2 \mathrm{~ms}$. In this work, we propose an experimental set-up to measure RTN that provides a higher time resolution than that available in standard characterization equipment. This new set-up, in combination with the Weighted-Time Lag (W-TL) method [17] for the analysis of the recorded signals, allows getting additional and relevant information about the RTN phenomenon, which cannot be obtained using standard characterization set-ups. Despite RTN signals can be observed in both resistance states (HRS [18] and LRS [19]), this work is focused on the analysis at HRS, because RTN is easier to be observed for large dielectric resistance values [20] with lower currents through the dielectric.

\section{MeAsurement Procedure}

The studied Ni/HfO2/Si devices were fabricated on (100) n-type CZ silicon wafers with resistivity between $0.007 \Omega \mathrm{cm}$ and $0.013 \Omega \mathrm{cm}[21]$. After standard wafer cleaning, a wet thermal oxidation process was done at $1100^{\circ} \mathrm{C}$ leading to a $200 \mathrm{~nm}$-thick $\mathrm{SiO} 2$ layer. 
This field oxide was patterned by photolithography and wet etching. Prior to the high-k deposition, a cleaning in $\mathrm{H} 2 \mathrm{O} 2 / \mathrm{H} 2 \mathrm{SO} 4$ and a dip in $\mathrm{HF}(5 \%)$ were performed. Subsequently, 20nm-thick HfO2 layers were grown by atomic layer deposition (ALD) using Tetrakis (dimethylamido)-hafnium (TDMAH) and $\mathrm{H} 2 \mathrm{O}$ as precursors, and N2 as carrier and purge gas. The deposition temperature was $225^{\circ} \mathrm{C}$. The top metal electrode, consisting of a 200nm-thick Ni layer, was deposited by magnetron sputtering. The resulting structures are square cells of $5 \times 5 \mu \mathrm{m} 2$. A schematic cross-section of the final device structure is shown in Fig. 1.

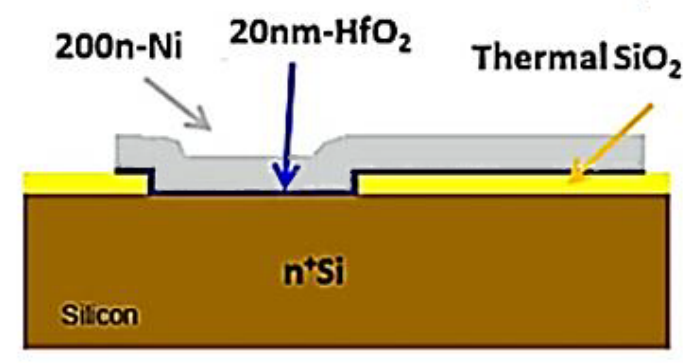

Fig. 1: Cross-section of the devices under test (DUT), where the different materials in the stack are indicated.

In order to get a stable $\mathrm{CF}$, the devices were subjected to 30 resistive switching (RS) cycles (where the first cycle corresponds to the forming process [6]), changing successively the dielectric conductivity between a high and a low resistance state, fixing a current limit of $10 \mu \mathrm{A}$ when switching to the LRS. Some RS cycles are shown in Fig 2. Set process, i.e. the transition from HRS to LRS (blue curves) and the reset process, i.e. the change from LRS to HRS (red curves) are observed. After this initial cycling, with the device at HRS, a RTN signal was sought by applying different voltage bias. When current fluctuations were detected, RTN measurements were carried out using a SPA and the new set-up. Fig. 3 shows a schematic of the experimental set-up developed to measure RTN with large temporal resolution. The voltage at which RTN appears, Vapp, was applied to one of the terminals of the DUT by the SPA (Agilent 4156C), which also 
allows measuring the current through this terminal. The other terminal of the DUT was connected to a logarithmic current-to-voltage converter (Log-IVC), which allows measuring currents that can differ in several orders of magnitude. The voltage from the current-to-voltage converter is derived to a digital storage oscilloscope (DSO, Tektronix TDS220) in order to register data at different time scales. This second DUT terminal is also connected to a high impedance buffer, so that a precise value of the voltage drop across the DUT can be measured. By changing the time scale of the DSO, the current through the DUT can be measured with different time resolutions, so that fast events can be captured. Though the oscilloscope can allow time resolutions in the nanosecond range, the limitation in the acquisition time would actually come from the circuit bandwidth.

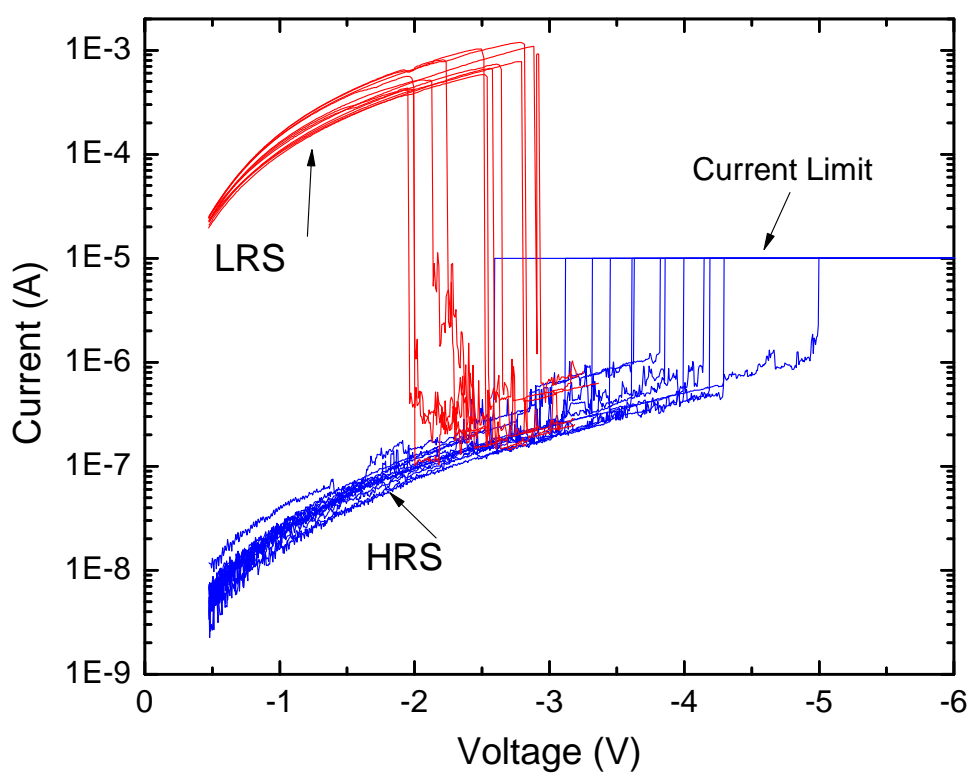

Fig 2: Initial RS cycling. Transition from HRS to LRS (blue lines) with current limit established at $10 \mu \mathrm{A}$ and transition from LRS to HRS (red lines). X axis corresponds to the voltage drop across the DUT, so that it does not start at 0 . 


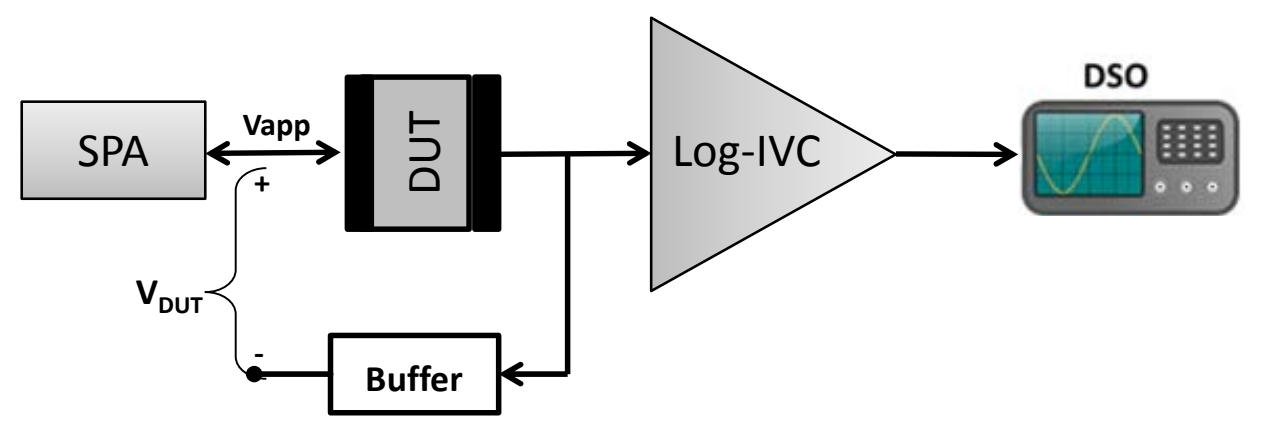

Fig. 3: Schematics of the experimental set-up developed to measure RTN signals with large time resolution. Vapp is the voltage applied by the semiconductor parameter analyzer and $\mathrm{V}_{\text {DUT }}$ is the voltage drop across the DUT.

Fig. 4 shows the block diagram of the RTN measurement process. First, RS cycling is performed to reach stable resistance states and then the suitable Vapp for RTN detection is chosen. After that, the HRS current is measured as a function of time. During the SPA measurement, several oscilloscope captures are registered using different time scales. When the SPA measurement finishes, the sequence defined in Fig. 4 starts again. In this work, about 325 SPA measurements were done and 21 oscilloscope captures were obtained for each SPA measurement.

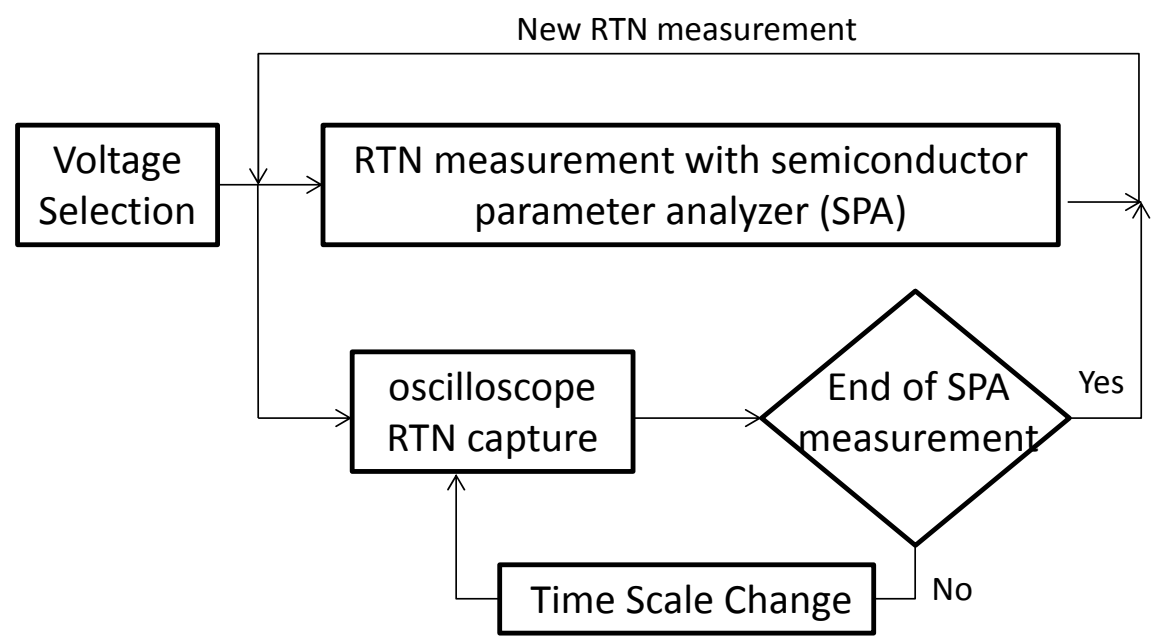

Fig. 4: Flux of the RTN measurement process once a RTN signal is detected at a particular voltage. RTN signals are simultaneously measured with the SPA and the developed set-up. The time scale of the DSO is changed during the measurement.

\section{AnAlysis Procedure}


Between the different methods to analyze and characterize RTN [22], Time Lag Plot (TLP)-based methods are the most common. A TLP is drawn by plotting the i-th point of the RTN signal in the x-axis and the (i+1)-th point in the y-axis for the full RTN trace [23]. Across the diagonal of the plot, every populated spot corresponds to one level of the RTN, whereas populated spots outside the diagonal correspond to transitions between states (Fig. 5).
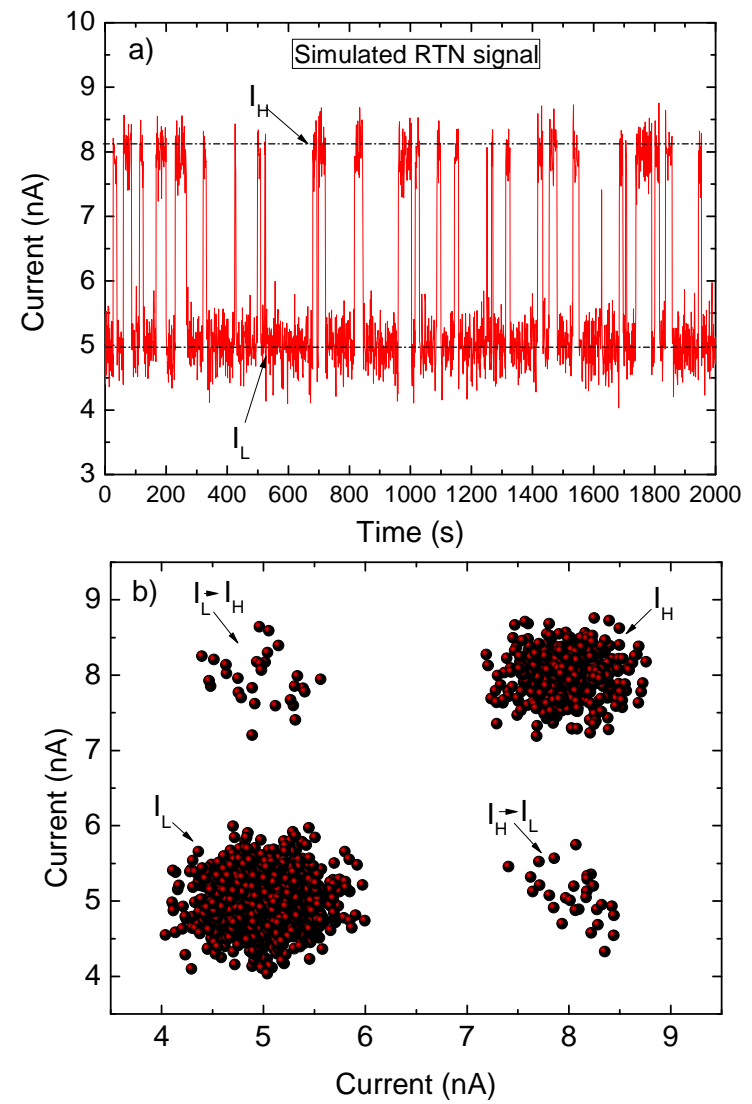

Fig. 5: (a) Simulated RTN signal caused by a single defect. (b) The corresponding time-lag plot: two populated spots on the main diagonal indicate the two states. Other clusters of points outside the diagonal correspond to transition between states.

TLP is useful when the RTN current jumps are well-defined or when different current levels are easy to identify at naked eyed, like in Fig. 5(b). However, when the background noise becomes relevant, different spots overlap each other, being difficult to separate the RTN current levels. Fig. 6(a) shows an example of a simulated RTN signal 
with large background noise. Applying TLP (Fig. 6(b)) it is not possible to distinguish any state or level so that RTN characterization becomes complicated. However, there is a new characterization method named Weighted Time Lag (W-TL) [17] which improves TLP. The W-TL method minimizes the effect of the background noise in the RTN signal allowing a more accurate analysis of the random telegraph parameters. Calculating a weighted function, which is based on a normal bivariate distribution of $\mathrm{i}$-th point and (i+1)-th point, on each dot in TLP a more accurate RTN analysis can be done. In Fig. 6(c) W-TLP of the RTN signal shown in Fig. 6(a) is depicted. Two states along the diagonal $(\Psi H, \Psi L)$ are revealed. These states were undetectable in the conventional TLP (Fig. 6(b)).

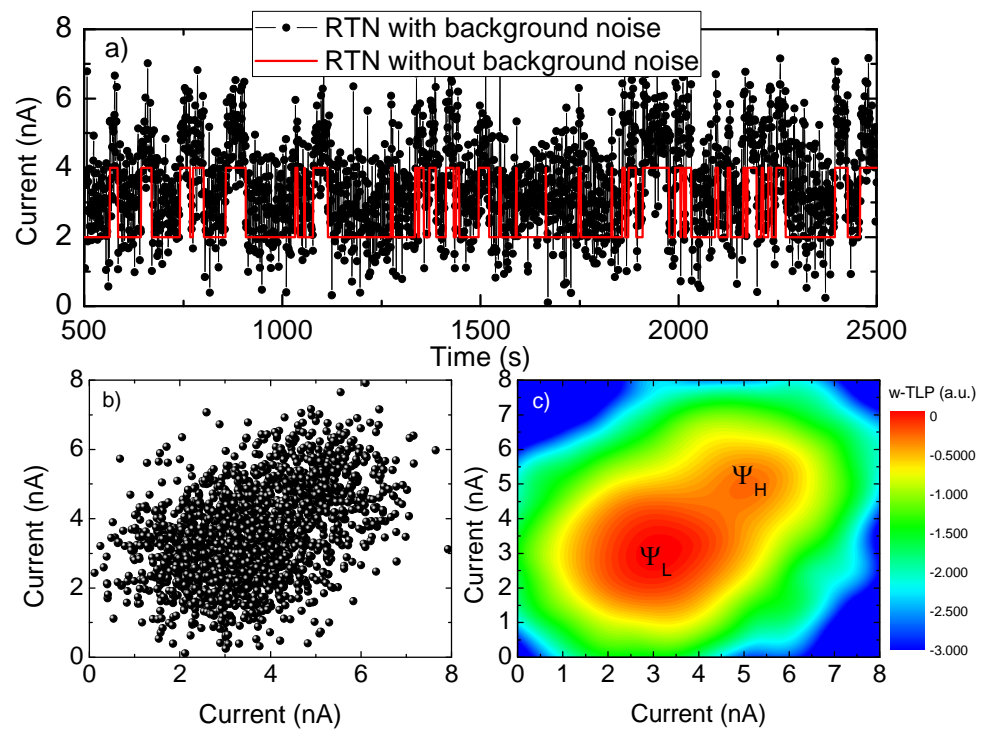

Fig. 6: (a) Simulated RTN signal with and without background noise; (b) conventional TLP of the RTN; the background noise hides the current levels and transition regions, (c) W-TLP where two states are encountered as red spots $(\Psi \mathrm{H}, \Psi \mathrm{L})$. [17]

The W-TL method is not only applicable to 2-levels RTN (Fig. 6) but also to multilevel RTN. In Fig 7(a), (b), and (c), an experimental multilevel RTN signal of a pMOS transistor, the conventional TLP and the W-TLP are shown respectively. In both Fig 7(b) and Fig. 7(c) the diagonal of the plot is identifiable. Regarding current states, with the 
TLP (Fig 7(b)) the states are more difficult to identify being only those located at the corners of the different originated squares clearly distinguished. However, there are some states that remain unclear due to overlapping or small quantity of dots. On the contrary, using W-TLP states that are not visible using conventional RTN characterization methods can be detected. Fig 7(c) represents W-TLP of the experimental multilevel RTN shown in Fig 7(a). Unlike TLP, this plot reveals up to 6 levels only looking at the plot.
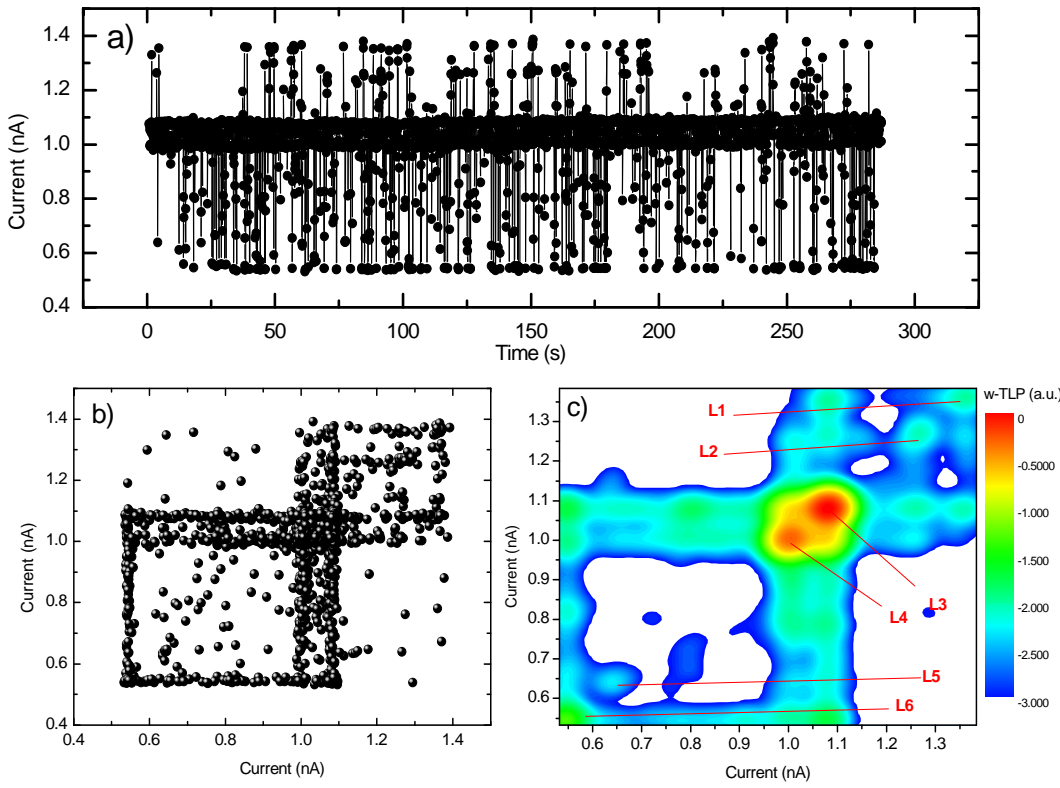

Fig. 7: (a) Experimental multilevel RTN in the gate current of a pMOS transistor, (b) Conventional TLP of a multilevel RTN signal, (c) W-TLP obtained from the RTN of (b). [17]

The W-TL method presents an additional advantage to find the RTN current states more accurately. W-TLP allows obtaining the maximum peaks of the diagonal which correspond to the different current levels of the RTN signal. Fig. 8 represents the diagonal peaks of the W-TLP shown in Fig 7(c). In the W-TLP, 6 states were identifiable but with the plot of the diagonal peaks two additional states (L7 and L8) are encountered. 


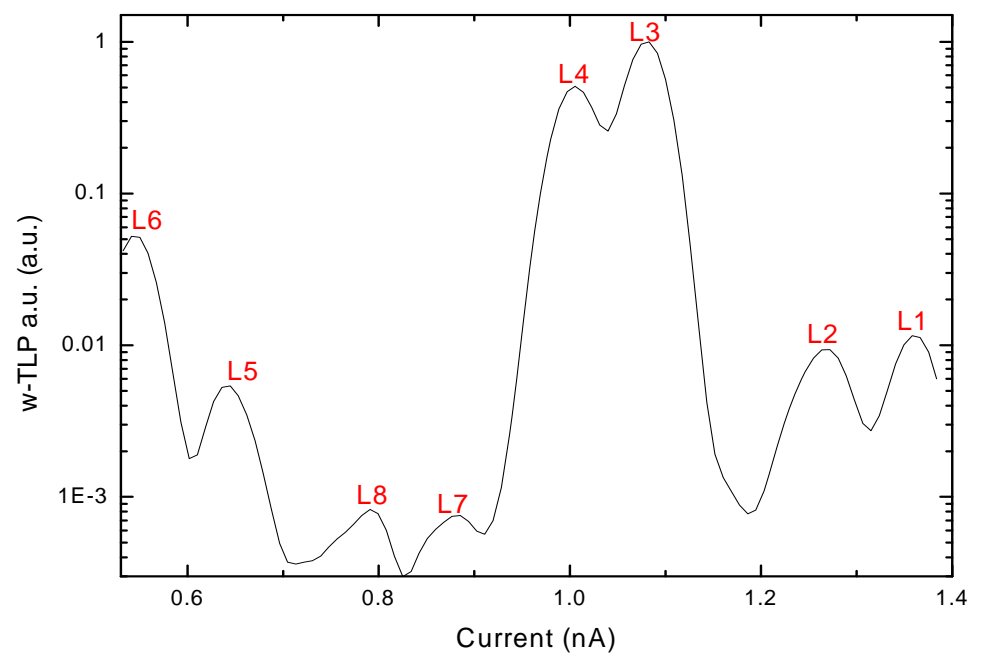

Fig. 8: Peaks of the diagonal of the W-TLP shown in Fig. 7(c). 8 peaks, i.e. 8 current states are encountered instead of the 6 states from W-TLP.

In summary, W-TL method allows analyzing RTN signals more accurately than standard TLP, being possible to distinguish more RTN current levels.

\section{Results}

As mentioned in the previous section, RTN signals are measured using two different systems, one of them based on a standard SPA and the other one based on an oscilloscope. In Fig. 9(a) a typical multilevel RTN signal measured by the SPA, with a time resolution of $6 \mathrm{~ms}$, is shown. At naked eye, 5 different current levels are evidenced. By applying the W-TL method to this RTN signal more levels are encountered concretely 9 different levels (L1-L9) (Fig. 9(b)). On the other hand, Fig. 10 shows three oscilloscope captures using time scales of (a) $40 \mu \mathrm{s}$, (b) $20 \mu \mathrm{s}$ and (c) $1 \mu \mathrm{s}$, i.e. different time resolution, recorded simultaneously to the SPA measurement in Fig. 9 at different time slots. Measurements at higher and lower time scales were done but only those at $40 \mu \mathrm{s}, 20 \mu \mathrm{s}$ and $1 \mu \mathrm{s}$ showed current fluctuations. These fluctuations on the traces correspond to some charge trapping and detrapping events in/from traps, responsible for 
the RTN signal and they appear with switching times lower than $6 \mathrm{~ms}$. These fast current fluctuations cannot be detected with the SPA. Fig. 10 shows some current levels which are coincident with those in Fig. 9(b) (L2, L3, L5, L6 and L7). However, in Fig. 10(b) and in Fig. 10(c) there is a new level (L10) that does not appear in Fig. 9(a), because of the fast transitions between states. Note that the setup allows measuring fast events, but that to determine the emission/capture times of the associated traps, a large amount of data would be needed, to carry out a statistical study.

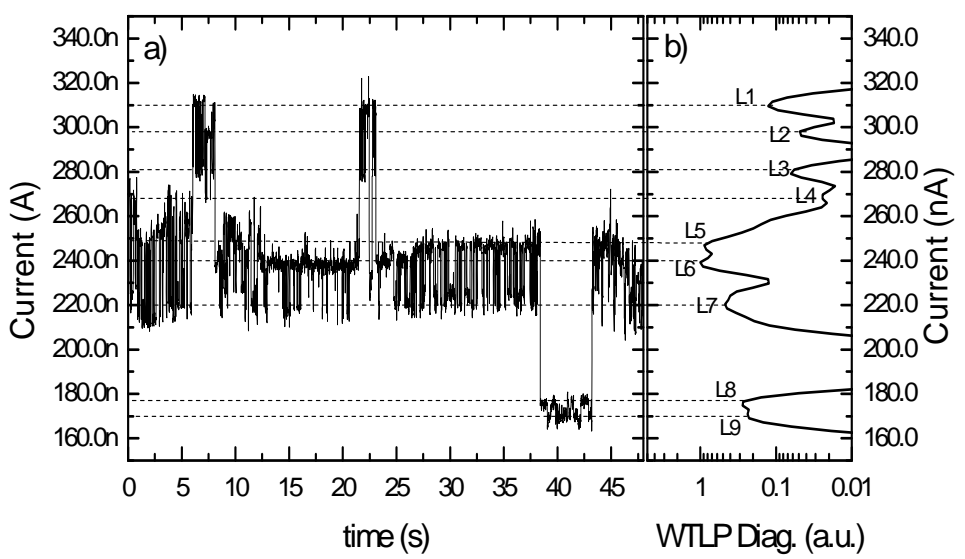

Fig. 9: (a) Typical multilevel RTN signal measured with a semiconductor parameter analyzer. Vapp $=1.25 \mathrm{~V}$, step time $\sim 6 \mathrm{~ms}$ and number of measured points 8000 . (b) Current levels obtained by using the W-TL method 

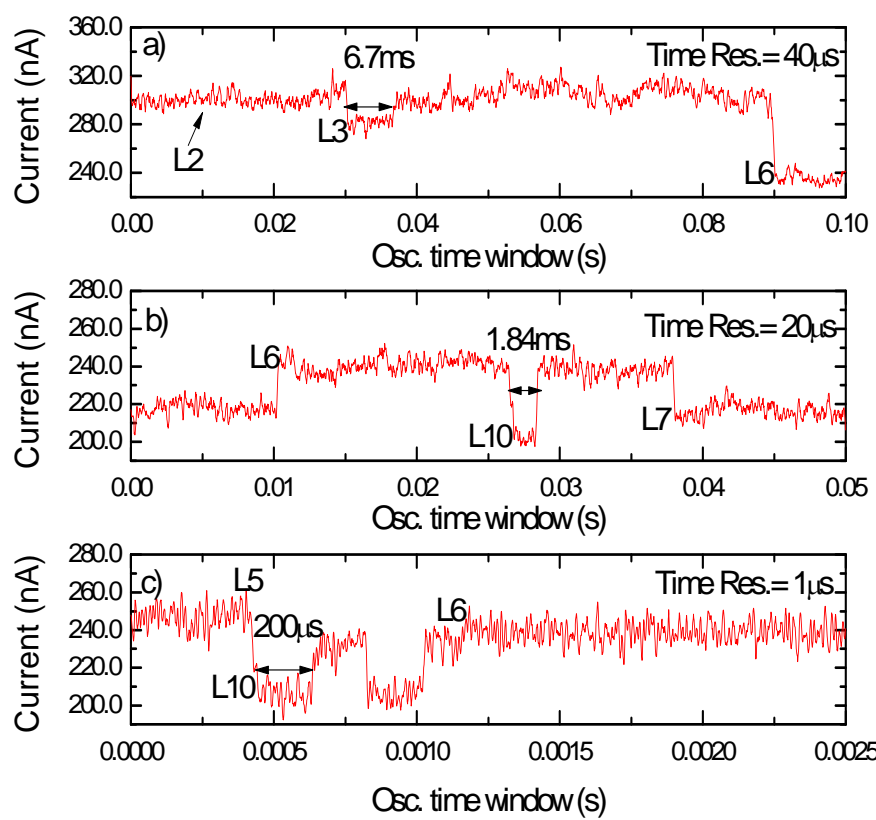

Fig. 10: Oscilloscope traces captured in the interval of: (a) 7.26s-9.68s of the SPA measurement shown in Fig. 9(a) with $40 \mu \mathrm{s}$ time resolution. (b) $9.68 \mathrm{~s}-12.15 \mathrm{~s}$, with a time resolution of $20 \mu \mathrm{s}$. (c) $19.5 \mathrm{~s}-21.93 \mathrm{~s}$ with a time resolution of $1 \mu \mathrm{s}$.

To complete the analysis and find more information about the current levels of the RTN trace shown in Fig. 9(a), the W-TL method was used. In Fig 11, W-TLP of the SPA measurement and of the three oscilloscope captures are obtained. Evaluating the diagonal of the plots resulting from the method and finding the maxima of that diagonal, all the levels in an RTN signal can be determined, since each of those maxima corresponds to a current (trap) level of the RTN. Fig 11(a) shows the W-TL plot of the whole RTN signal measured with the SPA presented in Fig. 9(a). A profile of the diagonal is plotted in Fig. 9(b) where all the detected levels are sequentially numbered. From the analysis of the WTL plot diagonal, 9 peaks and therefore 9 current levels (labelled as L1-L9) are obtained. Note that the number of current levels as determined by the W-TL method is larger than that encountered in the naked-eye analysis. The guide lines plotted in Fig. 9 help to observe a good match between the peaks in the diagonal of the W-TL plot and the current levels in Fig. 9(a). 
W-TL method was also used to analyze the RTN signals captured by the oscilloscope. Fig 11(b), (c) and (d) show the W-TL plots of these three captures and, as in the case of Fig 11(a), the existing traps levels are identified. Comparing these figures with Fig 11(a), we can recognize levels 2, 3, 5, 6 and 7. But from Fig 11(c) and (d), a new trap level at around 200nA, labelled as L10, appears, as mentioned before. Then, the presence of 10 current levels indicates, according to [23], at least four active traps contributing to the RTN fluctuations. This latter state does not appear in the SPA measurement because of the equipment resolution. However, the larger time resolution of the developed set-up highlights its presence and it can be clearly visualized in the corresponding W-TL plot. To study in detail the RTN levels found at different time resolutions the different W-TLP in Fig. 11 have been analyzed. Fig. 12(b), (c) and (d) show the diagonal profiles of the captures with $40 \mu \mathrm{s}, 20 \mu \mathrm{s}$ and $1 \mu \mathrm{s}$ time resolution respectively. SPA diagonal profile is also included for comparison in Fig. 12(a). Analyzing Fig. 12(b), (c) and (d) different peaks can be found. For a time resolution of $40 \mu \mathrm{s}$ (Fig. 12(b)), three peaks are encountered. From the center of the peaks the corresponding current states can be identified. In this case, these three peaks correspond to levels L2, L3 and L6. For time resolutions of $20 \mu \mathrm{s}$ (Fig. 12(c)) and $1 \mu \mathrm{s}$ (Fig. 12(d)) three different peaks are also encountered, and therefore three levels are evidenced. For $20 \mu$ s time resolution these levels are L6, L7 and L10 and for $1 \mu$ s L5, L6 and L10. All the peaks (i.e. current levels) encountered in these three plots are correlated with those in Fig. 12(a) with the exception of L10. The peak corresponding to this level appears at around 200nA in Fig. 12(c) and Fig. 12(d). In the diagonal profile of the SPA measurement no peak for current values around $200 \mathrm{nA}$ was found. This observation indicates that this current level was not possible to be measured with the SPA. 
The analysis performed from the diagonal profiles confirms the results obtained previously with W-TLPs. W-TL method can help to identify clearly some peaks that can be badly defined or unclear using the conventional TLP method.

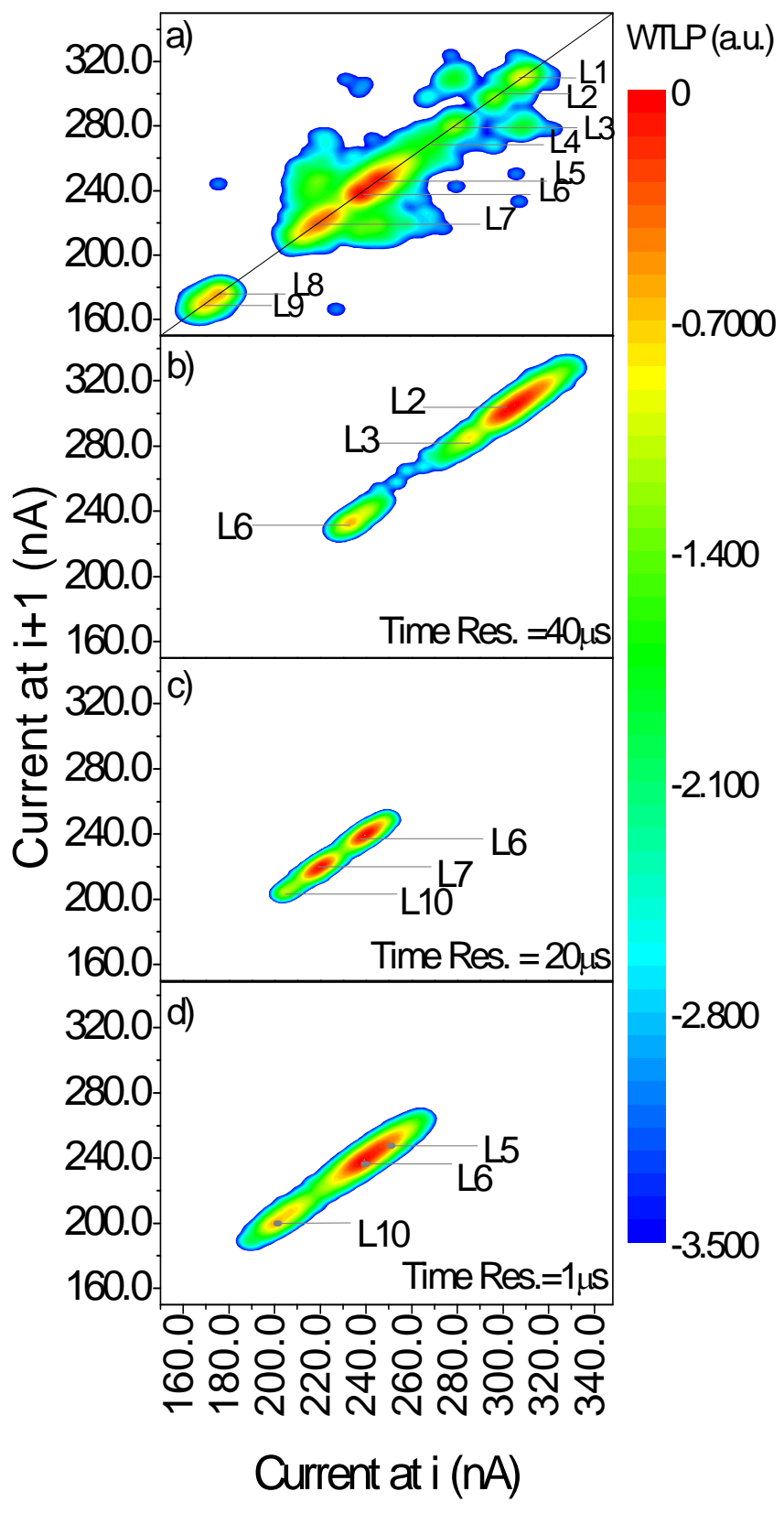

Fig. 11: W-TL method applied to(a) the RTN measured by the SPA (Fig. 9(a)). The line shows the diagonal of the plot, whose maximums correspond to the RTN current levels. 9 trap levels are detected; (b) oscilloscope capture with a time resolution of $40 \mu$ s (Fig. 10b), 3 trap levels appear; (c) oscilloscope capture with time resolution of $20 \mu \mathrm{s}$ with 3 trap levels (Fig. 10(c); (d)) oscilloscope capture at the larger time resolution $(1 \mu \mathrm{s})$, showing 3 trap levels (Fig. 10(d)). 

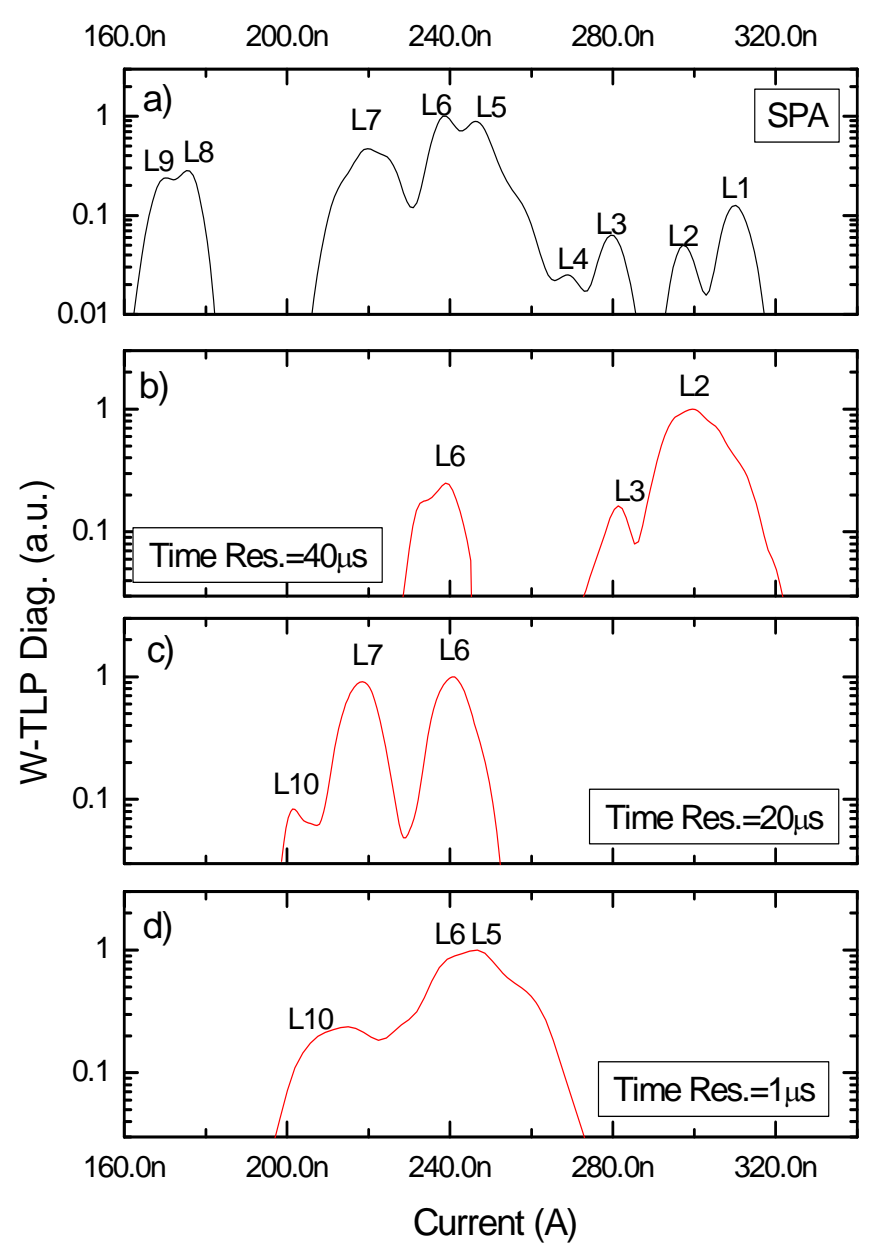

Fig. 12: Profiles of the W-TLP diagonal for (a) SPA measurement, where 9 peaks were encountered and for oscilloscope captures with time resolution of (b) $40 \mu \mathrm{s}$, with peaks corresponding to levels 6,3 and 2, (c) $20 \mu \mathrm{s}$, with levels 6, 7 and 10 and (d) $1 \mu \mathrm{s}$, with levels 5, 6 and 10 .

\section{CONCLUSIONS}

A new experimental set-up that provides a large time resolution is presented to analyze RTN signals. The results show that RTN fluctuations detected with the new set-up can be hidden if standard RTN characterization equipment as semiconductor parameters analyzers with lower time resolution is used. Moreover, when these RTN signals are analyzed with the W-TL method, the RTN levels can be accurately identified. Therefore, using our proposed characterization methodology, which combines high time resolution equipment and accurate parameters extraction, complete information about 
RTN can be obtained. This methodology allows a precise characterization of the RTN current fluctuations observed in RRAMs.

\section{Acknowledgement}

UAB authors acknowledge funding from the Spanish MINECO and ERDF (TEC201345638-C3-1-R) and the Generalitat de Catalunya (2014SGR-384). IMB-CNM authors thank the support of the Spanish MINECO under Project No. TEC2011-27292-C02-02.

\section{References}

[1] A. Sawa, "Resistive switching in transition metal oxides," Materials Today vol. 11, no. 6, pp. 28-36, 2008.

[2] R. Waser and M. Aono, "Nanoionics-based resistive switching Memories," Nature Materials, vol. 6, pp. 833-840, 2007.

[3] L. Baldi and G. Sandhu, "Emerging memories," in Proceedings of ESSDERC, pp. 30-36, 2013.

[4] H. Y. Lee, Y.-S. Chen, P. S. Chen, P. Y. Gu, Y. Y. Hsu, S. M. Wang, W. H. Liu, C. H. Tsai, S. S. Sheu, P.-C. Chiang, W. P. Lin, C.-H. Lin, W.-S. Chen, F. T. Chen, C. H. Lien, and M. Tsai, "Evidence and solution of over-RESET problem for HfOX based resistive memory with sub-ns switching speed and high endurance," in IEEE International Electron Devices Meeting (IEDM), pp. 19.7.1-19.7.4, 2010.

[5] A. C. Torrezan, J. P. Strachan, G. Medeiros-Ribeiro, and R. S. Williams, "Subnanosecond switching of a tantalum oxide memristor," Nanotechnology, vol. 22, p. 485203, 2011.

[6] H.-S. P. Wong, H.-Y. Lee, S. Yu, Y.-S. Chen, Y. Wu, P.-S. Chen, B. Lee, F. T. Chen, and M.-J. Tsai, "Metal-Oxide RRAM," Proceedings of the IEEE, vol. 100, no. 6, pp. 1951-1970, Jun. 2012.

[7] Y. Y. Chen, B. Govoreanu, L. Goux, R. Degraeve, A. Fantini, G. S. Kar, D. J. Wouters, G. Groeseneken, J. A. Kittl, M. Jurczak, and L. Altimime, "Balancing SET/RESET pulse for edurance in 1T1R bipolar RRAM," IEEE Transactions on Electron Devices, vol. 59, no. 12, pp. 3243-3249, Dec. 2012. 
[8] F. Nardi, D. Ielmini, C. Cagli, S. Spiga, M. Fanciulli, L. Goux, and D. J. Wouters, "Control of filament size and reduction of reset current below $10 \mu \mathrm{A}$ in $\mathrm{NiO}$ resistance switching memories," Solid-State Electronics, vol. 58, no. 1, pp. 42-47, Apr. 2011.

[9] N. Raghavan, R. Degraeve, L. Goux, A. Fantini, D.J. Wouters, G. Groeseneken and M. Jurczak, "RTN insight to filamentary instability and disturb immunity in ultralow power switching HfOx and AlOx RRAM", In 2013 Symposium, on VLSI technology (VLSIT), pp. T164-165, 2013.

[10] N. Raghavan, R. Degraeve, A. Fantini, L. Goux, S. Strangio, B. Govoreanu, D. Wouters, G. Groeseneken, and M. Jurczak, "Microscopic origin of random telegraph noise fluctuations in aggressively scaled rram and its impact on read disturb variability," IEEE International Reliability Physics Symposium (IRPS), pp. 5E.3.15E.3.7, 2013.

[11] S. Ambrogio, S. Balatti, A. Cubeta, A. Calderoni, N. Ramaswamy and D. Ielmini, "Statistical fluctuations in HfOx resistive-switching memory. part IIrandom telegraph noise ," IEEE transactions on Electron Devices, vol. 61, no. 8, pp. 2920-2927, 2014.

[12] S. Balatti, S. Ambrogio, A. Cubeta, A. Calderoni, N. Ramaswamy and D. Ielmini, "Voltage-dependent random telegraph noise (RTN) in HfOx resistive RAM," IEEE International Reliability Physiscs Symposium, pp. MY.4.1-MY.4.6, 2014.

[13] C.Monzio Compagnoni, R. Gusmeroli, A. S. Spinelli and A. Visconti, "RTN Vt instability from the stationary trap-filling condition: An analytical spectroscopi investigation,” IEEE Transaction Electronic Devices, vol. 55, pp. 655-661, 2008.

[14] D. Veksler, G. Bersuker, L. Vandelli, A. Padovani, L. Larcher, A. Muraviev, B. Chakrbarti, E. Vogel, D. C. Gilmer and P.D. Kirsch, "Random telegraph noise (RTN) in scaled RRAM devices," International Reliability Physics Symposium (IRPS), pp. 101-104, 2013.

[15] F.M. Puglisi, P. Pavan, A. Padovani and L. Larcher, "A study on HfO2 RRAM in HRS Based on I-V and RTN analysis," Solid- State Electronics, vol. 102, pp. 6975, 2014.

[16] F.M. Puglisi, P. Pavan, A. Padovani, L. Larcher and G. Bersuker, "RTS noise characterization of HfOx RRAM in high resistive state," Solid- State Electronics, vol. 84, pp. 160-166, 2012

[17] J. Martin-Martinez J. Diaz, R. Rodriguez, M- Nafria and X. Aymerich, "New weighted time lag method for the analysis of random telegraph signals," IEEE Electron Device Letters, vol. 35, no. 4, pp. 479-481, 2014. 
[18] N. Raghavan, R. Degraeve, A. Fantini, L. Goux, S. Strangio, B. Govoreanu, D. J. Wouters, G. Groeseneken, and M. Jurczak, "Microscopic origin of random telegraph noise fluctuations in aggressively scaled RRAM and its impact on read disturb variability," in IEEE International, Reliability Physics Symposium (IRPS), pp. 5E.3.1-5E.3.7, 2013.

[19] F. M. Puglisi, P. Pavan, L. Larcher, and A. Padovani, "Analysis of RTN and cycling variability in HfO2 RRAM devices in LRS," in Solid State Device Research Conference (ESSDERC), pp. 246-249, 2014.

[20] D. Ielmini, F. Nardi, and C. Cagli, "Resistance-dependent amplitude of random telegraph-signal noise in resistive switching memories," Applied Physics Letters, vol. 96, no. 5, pp. 053503_1-053503_3. 2010.

[21] M.B. Gonzalez, J. Rafi, O. Beldarrain, M. Zabala and F. Campabadal, “Analysis of the switching variability in Ni/HfO2- based RRAM devices," IEEE Transactions on Devices and Material Reliability, vol. 14, no. 2, pp. 769-771, 2014.

[22] L. R. Rabiner, "A tutorial on hidden markov models and selected applications in speech recognition”, Proceedings of the IEEE, vol. 77, pp. 257-285, 1989.

[23] T. Nagumo, K. Takeuchi, S. Yowogawa, K. Imai and Y. Hayashi, "New analysis methods for compehensive understanding of random telegraph noise," IEEE International Electron Devices Meeting (IEDM), pp. 1-4, 2009. 